\title{
BMJ Open How is defensive medicine understood and experienced in a primary care setting? A qualitative focus group study among Danish general practitioners
}

\author{
Elisabeth Assing Hvidt, ${ }^{1}$ Jesper Lykkegaard, ${ }^{1}$ Line Bjørnskov Pedersen, ${ }^{1,2}$ \\ Kjeld Møller Pedersen, ${ }^{3}$ Anders Munck, ${ }^{1}$ Merethe Kousgaard Andersen ${ }^{1}$
}

To cite: Assing Hvidt $\mathrm{E}$, Lykkegaard J, Pedersen LB, et al. How is defensive medicine understood and experienced in a primary care setting? A qualitative focus group study among Danish general practitioners. BMJ Open 2017;7:e019851. doi:10.1136/ bmjopen-2017-019851

- Prepublication history for this paper is available online. To view these files, please visit the journal online (http://dx.doi org/10.1136/bmjopen-2017019851).

Received 29 September 2017 Revised 17 November 2017 Accepted 20 November 2017

CrossMark

${ }^{1}$ Department of Public Health, Research Unit of General Practice, University of Southern Denmark, Odense, Denmark

${ }^{2}$ Department of of Business and Economics, COHERE, University of Southern Denmark, Odense, Denmark

${ }^{3}$ Department of of Business and Economics, COHERE, University of Southern Denmark, Odense, Denmark

Correspondence to Dr Elisabeth Assing Hvidt; ehvidt@health.sdu.dk

\section{ABSTRACT}

Objectives Recent years have witnessed a progressive increase in defensive medicine (DM) in several Western welfare countries. In Danish primary and secondary care, documentation on the extent of DM is lacking. Before investigating the extent of DM, we wanted to explore how the phenomenon is understood and experienced in the context of general practice in Denmark. The objective of the study was to describe the phenomenon of DM as understood and experienced by Danish general practitioners (GPS).

Design A qualitative methodology was employed and data were generated through six focus group interviews with three to eight GPs per group $(n=28)$ recruited from the Region of Southern Denmark. Data were analysed using a thematic content analysis inspired by a hermeneuticphenomenological focus on understanding and meaning.

Results DM is understood as unnecessary and meaningless medical actions, carried out mainly because of external demands that run counter to the GP's professionalism. Several sources of pressure to act defensively were identified by the GPs: the system's pressure to meet external regulations, demands from consumerist patients and a culture among GPs and peers of infallibility and zero-risk tolerance.

Conclusions GPs understand DM as unnecessary and meaningless actions driven by external demands instead of a focus on the patient's problem. GPs consider defensive actions to be carried out as a result of succumbing to various sources of pressure deriving from the system, the patients, the GPs themselves and peers.

\section{INTRODUCTION}

Rapid developments in medical technology, increases in medical expertise together with societal changes have contributed to several beneficial changes in the healthcare sector, for example, sophisticated diagnostic and treatment procedures and a less authoritative doctor-patient relationship. ${ }^{1}$ However, recent years' medical developments have also promoted a culture in which high expectations for diagnosing, treatment and cure encourage health service users to sue

\section{Strengths and limitations of this study}

- Employing a qualitative methodology eliciting discussion and reflection among general practitioners (GPS), we have been able to achieve a nuanced understanding of defensive medicine (DM) that is closely connected to the everyday experiences, routines, activities and views of GPs in relation to $\mathrm{DM}$.

- Whereas it is beyond the methodological scope of this study to claim empirical generalisability, the research findings are transferable to other GPs, physicians and health professionals from similar cultural and organisational contexts and with countries with similar institutional, legal and medical systems.

- Further validity and depth could have been added to the study if additional individual interviews with the participating GPs had been conducted subsequently, making it possible to deepen some of the themes on an individual basis and to shed light on possible information bias resulting from lacking confidence in a group.

for malpractice or lodge formal complaints to health authorities, hereby encouraging physicians to practice defensively. ${ }^{2}$ Defensive medicine $(\mathrm{DM})$ is commonly defined as a deviation from standard medical practice due to fear of malpractice liability claims. ${ }^{13}$ The deviating medical practice may include two types of behaviour: an 'assurance behaviour' involving the ordering of more tests and procedures than medically indicated and an 'avoidance behaviour' in which the physician avoids high-risk procedures and/or patients to distance him/herself from malpractice liability. ${ }^{45}$

Many scholars claim DM to be a disadvantageous phenomenon, arguing that practising DM can be directly harmful to the patient (leading to fear and overtreatment), ${ }^{6}$ to 
society (entailing unwarranted use of resources) and to physicians (fear of being sued) ${ }^{78}$

Investigating the prevalence of DM in a number of international secondary healthcare settings, DM has been found to be highly prevalent in countries such as the USA, ${ }^{5}{ }^{10}$ Israel, ${ }^{11}$ Japan, ${ }^{12}$ Australia ${ }^{13}$ and within a European setting, in the UK, ${ }^{14}$ Italy $^{24}$ and Belgium. ${ }^{2}$ As for the prevalence of DM in a primary care setting, a study examining defensive medical practices in primary care in the USA showed that almost all general practitioners (GPs) acknowledged practice changes in response to the possibility of a patient complaint. ${ }^{8}$ Specific widespread practices were diagnostic tests, referrals and follow-ups as well as unnecessary medical records. A more recent American study revealed that among specialty groups, primary care physicians contributed the most to DM spending. ${ }^{15}$

In Denmark, documentation on the extent of DM in general practice as well as in the hospital sector is lacking. Danish physicians are not covered by the culpa legislation, meaning that they cannot be held financially liable for malpractice which instead is covered by the publicly financed Patient Compensation programme-a comprehensive national programme to compensate for patient harm. However, physicians may be sued individually with reference to the Physicians' Act Law (gross negligence) where the maximum penalty is losing their license to practice medicine or fines. ${ }^{16}$

Little is known about how GPs perceive of DM in a Danish primary care setting and which specific aspects motivate them to practice defensively.

Thus, the aim of this study was to identify individual and shared perspectives among GPs on how DM is understood and experienced in their daily clinical work.

\section{METHOD}

The methodological approach employed was rooted in a qualitative description inspired by a hermeneutic-phenomenological research methodology. ${ }^{17}$ As a method for data generation, focus group interviews were chosen because their interactional features were fit for exploring subjective understandings, experiences and viewpoints. ${ }^{1819}$

\section{Setting}

The Danish healthcare system is tax financed, and most GP and hospital services are free of charge. Danish GPs act as gatekeepers for access to specialist treatment and are responsible for front-line care 24 hours a day. GP collaborations provide out-of-hours primary care in four out of the five regions. ${ }^{20}$

\section{Recruitment and sample size}

GPs from one of Denmark's five regions with a minimum of 2 years of experience in general practice were invited to participate in a focus group interview. Research colleagues, not involved in the study and being practising GPs themselves, helped identify participants by providing email addresses to primary care clinics. We attempted to achieve variation with respect to age, gender, practice type, practice experience and practice location (rural or urban area). The final purposive sample comprised 28 GPs (14 men and 14 women) between 36 and 68 years of age (see table 1 for participant characteristics). All participants gave their written informed consent.

\section{Data generation}

Six focus group interviews (with three to eight participants per group) were held between October 2016 and May 2017. The first author, a sociologist and an experienced qualitative researcher, moderated all six groups and had neither professional knowledge of nor experience with DM. The last author, a researcher and practising GP, acted as comoderator in five out of six focus groups. Both researchers consciously and continuously explored their prejudgements about the phenomenon and wrote down field notes during or after each interview. The interviews were conducted in the office of one of the

\begin{tabular}{|c|c|c|c|c|c|c|}
\hline Focus groups $n=28$ (14 men; 14 women) & 1 & 2 & 3 & 4 & 5 & 6 \\
\hline Age range & $42-58$ & $40-52$ & $54-55$ & $46-52$ & $64-69$ & $30-68$ \\
\hline Mean & 45 & 46 & 54 & 50 & 67 & 45 \\
\hline \multicolumn{7}{|l|}{ GP practice type } \\
\hline Group (two or more GPs): G (N) & $\mathrm{G}(8)$ & G (3) & $\mathrm{G}(3)$ & G (4) & $\mathrm{G}(4)$ & G (5) \\
\hline $\mathrm{SH}(n)$ & & & & & $1(\mathrm{SH})$ & \\
\hline \multicolumn{7}{|l|}{ Practice location } \\
\hline$U(n)$ & $\cup(7)$ & & $U(1)$ & $\cup(4)$ & $U(2)$ & U (5) \\
\hline $\mathrm{R}(\mathrm{n})$ & $\mathrm{R}(1)$ & $\mathrm{R}(3)$ & $\mathrm{R}(2)$ & & $\mathrm{R}(3)$ & \\
\hline $\operatorname{Man}(n)$ & 0 & 2 & 0 & 4 & 5 & 3 \\
\hline Woman (n) & 8 & 1 & 3 & 0 & 0 & 2 \\
\hline Total (n) & 8 & 3 & 3 & 4 & 5 & 5 \\
\hline
\end{tabular}

GP, general practitioner; R, rural; $\mathrm{SH}$, single handed; U, urban. 
group informants (three groups) at a regional meeting room (two groups) or in the private home of one of the informants (one group). To facilitate a gradual disclosure of the GPs' understandings and experiences as they related to DM, we followed a semistructured interview guide with open-ended questions (table 2). Each focus group interview was initiated with a presentation of the explorative aim of the study, namely to capture individual and shared understandings of and experiences with DM as they related to daily clinical practice. Consequently, no formal definition of DM was presented. The recruitment of new groups continued until sufficient information power regarding the subject at hand was achieved. ${ }^{21}$ The discussions lasted from 1 hour to $75 \mathrm{~min}$ and were all digitally recorded, then transcribed verbatim by a secretary and validated by the researchers who moderated the interviews.

\section{Data analysis}

Data were analysed according to the core principles of a thematic analysis approach inspired by a hermeneutic-phenomenological focus on understanding and meaning. ${ }^{22}$ The first and last authors (EAH and MKA) performed the analysis. The continuous analytic process, with description of coding themes, was presented to and discussed with the other members of the author group at regular analytical meetings. The analytical process moved through the following stages: interview transcripts were read in their entirety several times to gain a general understanding of the data. The text was divided into meaning units that were grounded in the particularity of what was being said by the participants. ${ }^{22}$ The subsequent stage of analysis aimed at transforming meaning units into larger themes with special attention to how they related to the research questions. Significant meaning units documenting participants' understandings of and experiences with DM were categorised. Some of the meaning units were found to be replete with utterances that described experiences of pressure. These utterances were categorised into different types of pressure. We acknowledge that they cannot be considered exhaustive and may overlap. In the following, the key themes and subthemes are presented with exemplary data sequences.

\section{RESULTS}

\section{Theme 1: GPs' understanding of DM}

In most focus groups, GPs were quick to respond to the question about what they understood by the phenomenon of DM. With few variations, GPs stated that they understood DM as medical actions performed without medical indication to 'cover one's back' and to secure oneself against patient complaints. Interestingly, however, when exploring and discussing the phenomenon of DM more in depth, several of the GPs found that this understanding was not sufficiently comprehensive when considering the plethora of daily defensive actions in general practice. Across groups, understandings of DM were broadened to involve all those unnecessary and meaningless medical actions performed due to external demands that run counter to the GP's professionalism and common sense. For example, one of the GPs remarked:

FG5GP5: One tends to immediately think that it's something we do to protect ourselves against patient claims, right, but in reality, maybe it's more like the sum of unnecessary actions that makes it a little exhausting to be a GP?!

Table 2 Interview guide for the focus group interviews

\begin{tabular}{|c|c|}
\hline Main themes & Probing questions \\
\hline $\begin{array}{l}\text { Understandings } \\
\text { of DM }\end{array}$ & What do you at first understand by the concept 'Defensive medicine' when you hear it? \\
\hline Motives & $\begin{array}{l}\text { Now that you have listened to each other you might recognise some features and situations from your own } \\
\text { practice. If you again recall the specific situation, which you have described, what do you think was the } \\
\text { reason(s) for acting as you did? }\end{array}$ \\
\hline Perceptions & $\begin{array}{l}\text { Can you try to describe how you perceived these situations? } \\
\text { What kind of feelings did they initiate (if any)? } \\
\text { To what extent do these types of situations fill your mind? } \\
\text { How often do these types of consultations occur in your daily practice? (eg, never, seldom, often?) } \\
\text { If you look back in time, do you think you would have acted differently } 10 \text { years ago? }\end{array}$ \\
\hline Perspective & $\begin{array}{l}\text { If we look back on what we have talked about until now, do you have the same understanding of the } \\
\text { concept 'DM' as when we started out discussing it? }\end{array}$ \\
\hline
\end{tabular}

DM, defensive medicine. 
Extending the above understanding of DM, several of the GPs described DM as practices that one does as a result of pressure from something or somebody. One male GP described the feeling of being pressured in the following way:

FG4GP2: You are defending yourself against something, and I can think of many I must defend myself against. Must I defend myself against the patients? Must I defend myself against the medical officer of health? Must I defend myself against my colleagues? Must I defend myself against my own medical conscience? So, there are many things one can defend oneself against, and in this way, I think the concept can take up much space in everyday life!

Resonating with the above account, other GPs across groups consistently talked about how they experienced that DM as it unfolded in daily clinical practice resulted from daily pressures. In the following section, these different experiences of pressure that motivated the GPs to practice defensively will be outlined.

\section{Theme 2: GPs' own experiences with DM}

\section{Subtheme: system pressure}

A majority of the GPs talked about how 'the system', in many cases personified by the politicians and health authorities, pressured them to practice DM. These practices resulted from the system-imposed demand to comply and implement evidence-based standardised care such as clinical guidelines, fast-track packages (eg, cancer packages) and treatment guarantees. According to the GPs, these imperatives often resulted in 'thin' or 'non-sense' referrals. These actions were considered to be defensive because they were more substantiated by a pressure to live up to political regulations and time warrants than to meaningful clinical decision-making.

The experience among several of the GPs was that the obligation to comply with and implement clinical guidelines and refer patients to fast-track packages was undermining the individual GP's clinical assessment and professionalism:

FG4GP4: Society dictates that we must act on specific symptoms in such a way that we actually put aside our own professionalism....and so our professionalism is not in great demand any longer.

In relation to this, some GPs experienced that the national clinical guidelines were often not in accordance with their own clinical reality, despite being allegedly evidence based. Practising defensively by applying the guidelines without reflecting on their meaningfulness and thus pushing patients into rigid structures would, according to several of the GPs, too often do harm to the patients, for example, by leading to anxiety and overtreatment. Along these lines, other GPs said that acting defensively reflected a 'zero-tolerance culture':
FG4GP3: So we are asked to be very defensive, not to defend, or not to protect ourselves, but because society has decided that we cannot live with the teenyweeny risk that somebody calls the doctor and is told to take a pain killer and it turns out that they have a brain tumor or something, and I think that with this decision we shoot completely above the target!

Another recurring theme when reflecting on own experiences with DM was the demand to document (what some of the GPs described as 'limitless, meaningless documentation') that the government policy had imposed on the GPs for quality appraisal purposes. One practice that was particularly described as defensive by the GPs was the documentation of patient records involving long enumerations of negative clinical findings:

FG1GP1: For example our patient records, all the time we must write, this you didn't find, well, all the negative findings, there wasn't this, there wasn't this, there wasn't this... just think about the amount of resources that are spent on not having trust in professionals and all the time we have to beware, beware, beware, document, document, document!

When talking about how the tendency to document had increased in recent years, some of the GPs characterised the patient record as 'word salad' and 'spam' paradoxically compromising the quality of care and patient safety. To further illustrate this point, one male GP even brought a print of a patient's medical record, displaying the progression in note length over the past 5 years while uttering:

FG4GP2: Patient records just get longer and longer. The clarity and the details are lost and the patient trajectories almost drown in documentation.

\section{Subtheme: patient pressure}

All participating GPs talked about how they felt pressured to act defensively because of an increasing request from patients for medical examinations and referrals to specialists, leaving the GP with the impression that generally and compared with earlier, patients lack confidence in the clinical assessment of today's GPs. Across groups the GPs agreed that the socioeconomically privileged patients constituted a particularly demanding patient category:

FG1GP3: Generally, it's the kind of people who are well functioning who have the capacities to operate within this system and who have the resources to turn up at the doctor's office and put their foot down and demand to be given this or that, right? And it's not always those who really need the examinations that get through, is it?

FG1GP6: Nope, it's not social classes five-seven, definitely not!

Patients holding supplemental private health insurances were in particular experienced to exert pressure in 
that their insurance company had given them the prospect of a private treatment provided that their GP would refer them to these further examinations:

FG1GP6: Private health insurances are a substantial factor. Yes, there we are under great pressure, because their health insurance company has held out the prospect that they can be seen at a private hospital within a few days and they can have a scan. 'You just need a referral from your GP'. We hear that SO often.

Furthermore, the group of psychosomatic patients was by several of the GPs mentioned as a source of patient pressure:

FG3GP2: I think that our psychosomatic patients are probably the group of patients that pressure us the most to do the strangest things and afterwards one thinks: 'Come on! Why on earth did I agree to give that referral for this completely unnecessary examination?'

The GPs agreed that resisting patient pressure was further complicated and challenged by the dominating influence of the media. Several GPs pointed out that although increase in health education is generally a positive development, the health warnings communicated through the media, sometimes based on dubious scientific evidence, result in patients becoming increasingly fearful and anxious about risk factors and alarm symptoms, motivating them to request for specific tests and examinations.

The increase in patient complaints was also considered to be a result of the mass media's exposure of single stories of incompetent physicians and making people conscious of their 'rights', for example, to treatment guarantees, to complain/sue for malpractice with the prospect of receiving compensation.

\section{Subtheme: self-pressure}

The GPs acknowledged that a pressure deriving from themselves contributed to the increase in defensive medical actions, making some of the GPs voice that 'we are our own worst enemy'. One substantial pressure was described as the fear of making errors of judgement having lethal consequences for the patient. A way of minimising this fear in the daily work would be to reduce medical uncertainty to the lowest possible level by ordering further tests and examinations:

FG3GP1: Just overlooking something that has disastrous consequences for another human beingit does not even have to elicit a complaint, but just the risk of overlooking something, I mean that is terrible!

FG3GP2: Yes, then I'd rather play it safe.

FG3GP1: Yes, but this has nothing to do with the complaints!

As indicated in the above excerpt, the patient complaint as such, which a medical error might elicit, was perceived as secondary compared with the anguish of harming the patient. A culture of infallibility among GPs, in the medical community and in society at large, was highlighted by several of the GPs as maintaining their fear and thus as pressuring them to act defensively.

Every GP had experienced being either a subject or cosubject of a patient complaint at some stage in their career, not least when working in the out-of-hours primary care service. The patient complaints referring to these out-of-hours consultations were referred to as unjustified or ridiculous. The GPs explained that in the out-of-hours primary care service the relational bonds between GP and patients were weak and consequently, the threshold for complaints particularly low. Generally, the younger doctors were more concerned about receiving a patient complaint than the more experienced GPs.

\section{Subtheme: peer pressure}

Fear of having one's reputation damaged by colleagues was also perceived as a pressure that could motivate the GPs to perform defensive medical actions. Some of the GPs had experienced malicious statements and gossip by hospital colleagues following a medical error:

FG2GP2: And we have seen how easy it is to have two colleagues stand up together and state that the colleague who has made the error must be completely at sixes and sevens, right? Total stupid decision, how on earth could this happen?

Other GPs described how they felt pressured to perform a lot of examinations prior to hospitalising a patient, because they had experienced that the hospital physicians demanded as thorough examinations of the patient as possible:

FG2GP4: I mean, they stand there laughing at us when we call from the emergency service and we want to hospitalise somebody: 'No, you can't just do that without measuring both this and that and without having a broad blood picture and having cultivated the blood and x-raying this and x-raying that.'

Another kind of pressure deriving from colleagues or peers was the pressure to refer patients for scans or other examinations because other practitioners, for example, physiotherapists or chiropractors, were requesting examinations rather than the GP's assessment. Since the practitioner had already held out prospects of a particular examination to the patient, the GPs experienced the situation as involving a conflict that in most cases would result in giving in to the pressure of the practitioner's request:

FG3GP1: It gets really difficult when they have already written down their suggestions for further diagnosing and then the patient is already expecting you to refer for further diagnostics - then we are kind of checkmate!

FG1GP3: And I mean, this is really problematic because this is not what our guidelines tell us to do, 
but we can end up acting as defensively as ordering an MR scan after all.

\section{DISCUSSION}

\section{Summary}

In this study, we explored GPs' understandings of and experiences with DM. We found that GPs in a Danish general practice setting understand DM as unnecessary and meaningless medical actions. Drawing on their daily experiences the GPs furthermore reasoned that these defensive actions are carried out as a result of succumbing to daily pressures deriving from four different sources: the system, patients, the GPs themselves and colleagues.

\section{Comparison with existing literature}

American and European literature on DM focuses mainly on DM as medical behaviour (either assurance or avoidance behaviour) that follows from malpractice concerns. ${ }^{23} 24$ Although complaints constitute a shared concern among the GPs of this study, other forms of pressure appear to motivate a medical behaviour that is experienced as defensive. Our research thus documents that Danish GPs understand DM in a broader and more differentiated way than how the phenomenon has predominantly been defined within the health economical and judicial literature. We assert that if other GPs, physicians and health professionals from similar cultural and organisational contexts understand and experience DM this way then the research findings of this study complement the traditional definition of DM.

Supporting the finding that changes in medical behaviour are caused by malpractice concerns and even more pervasively, by externally imposed system pressures, the sociological literature argues that recent changes and reforms to which general practice has been subject, such as an increase in external accountability, monitoring and managerial controls as well as the movement towards evidence-based medicine as the dominant rationale for choice of treatment, represent a trend towards disciplining GP behaviour, hereby undermining their autonomy and authority. ${ }^{25-27}$ Seen from this perspective, the GP-perceived system pressures identified in this study might reflect these larger managerial processes in the healthcare system that the GPs experience as indirectly pressuring them to act defensively.

In line with the understanding of DM as unnecessary and meaningless medical actions, studies investigating GPs' emotional responses to their work in general practice find that medical actions in which the GPs' identity, professionalism and clinical judgement are compromised are experienced as meaningless. ${ }^{25} 27$ It is argued that a healthcare system emphasising standardised biomedical evidence-based practice, based on protocols and guidelines as a means to improving population rather than individual health, pays little attention to the context in which primary care consultations take place. The exceptional potential of the primary care consultation is said to include the continuing and personal GP-patient relationship, a multidimensional approach to illness (biopsychosocial) and person-centred medicine. ${ }^{28-30}$

As we have seen in the above, many GPs changed their professionally informed behaviours to adapt to the pressures coming from insistent 'consumerist' patients insisting on patient rights. Research has described the impact of an increasing consumerist 'ethos' in society in which medical professional knowledge is made available to lay people, mainly through the mass media, hereby challenging the medical dominance of the past as well as the professional identity of doctors-and ultimately quality of care. ${ }^{31} 32$ The result showing that the well-educated, articulated and young patients with minor health problems constituted a particularly demanding patient group is in line with research showing that consumerism and decreasing patient deference to physicians are influenced by factors such as age, education and by the seriousness of the illness. ${ }^{32}$ Furthermore, our finding that GPs feel pressured to act defensively by patients holding supplemental health insurances is supported by results from a recent Danish study showing that a majority of the 2000 surveyed GPs perceived this patient group as particularly insistent in getting referrals, and that almost half of the surveyed GPs felt a pressure to refer even when short of a medical indication. ${ }^{33}$

Relating to the subtheme of 'self pressure', physicians' sensitivity to the existential uncertainty of medicine and their concerns about the scope of error is a well-known research theme. ${ }^{25}{ }^{34}$ Furthermore, a vast body of literature describes the emotional impact of mistakes, for example, how making medical errors affects physicians unfavourably, creating a strong need for support within the medical community. ${ }^{25} 34-37$ As the findings of this study demonstrate, support from colleagues in the medical community is sometimes lacking, making the pressure to act defensively even bigger. Relating to this experience, a qualitative study investigating the views of doctors on their working lives found that physicians' feelings of nostalgia for the past were mainly connected to a loss of opportunities of informal mutual support between colleagues. ${ }^{25}$ These findings highlight the need for enhancing a supportive organisational climate and for encouraging interdisciplinary collaboration on reducing DM.

In $2000, \mathrm{Wu}^{38}$ introduced the definition of 'second victim', meaning that not only patients and relatives may be deeply disturbed by the errors and mistakes made by health professionals. ${ }^{39}$ From this perspective, it can be argued that the GPs of today's medical culture may live an increased risk of becoming 'second victims' following burdensome complaints and as a result of a daily clinical reality in which feelings of pressure from several sources dominate, hereby compromising professional identity, values and ideals.

\section{Implications for practice and research}

Our findings may lead to discussions within the medical establishment about the potential impact of externally 
imposed policy interventions on GPs' professional autonomy and sustainability of their work. Our findings indicate that DM will not be reduced without fundamental changes in the dominating cultures surrounding modern medical practice. Awareness of an increasingly defensive medical practice culture and its negative implications has paved the way for a much needed political focus, like the 'Choosing Wisely' campaign in the UK launched by the Academy of Medical Royal Colleges last year listing 40 tests and treatments that are unlikely to benefit patients, now being adopted to a Danish setting. ${ }^{40}$ Supplementing such campaigns, it may be of benefit to create alternative solutions to re-establish reflexivity in the medical community concerning matters such as core values and ideals regarding professional identity. However, as this study shows, 'choosing wisely' is not a 'free choice' but involves a support to the physician from for example, the professional organisation and moreover time and conditions for discussions with the patients regarding pros and cons for an intervention.

Future research should aim at estimating the costs of DM in primary care regarding implications for quality of care, professional motivation and satisfaction, time as well as monetary costs.

Acknowledgements The authors wish to thank all the GPs participating in this study for their time and interest.

Contributors EAH, JL, LBP, KMP, AM and MKA were involved in study conception and design. EAH and MKA were involved in acquisition of data. EAH, JL, LBP, KMP, AM and MKA were involved in analysis and interpretation of data. EAH and MKA were involved in drafting of manuscript. JL, LBP, KMP and AM were involved in critical revision of the manuscript.

Funding This project was funded by the Committee of Quality and Continuing Education Region of Southern Denmark (Kvali-projekt 07/16) (grant number: 16/16269).

Competing interests None declared.

Ethics approval Ethics approval was granted by The Danish Data Protection Agency (J. number: 16/46654)

Provenance and peer review Not commissioned; externally peer reviewed. Data sharing statement No additional data are available.

Open Access This is an Open Access article distributed in accordance with the Creative Commons Attribution Non Commercial (CC BY-NC 4.0) license, which permits others to distribute, remix, adapt, build upon this work non-commercially, and license their derivative works on different terms, provided the original work is properly cited and the use is non-commercial. See: http://creativecommons.org/ licenses/by-nc/4.0/

(C) Article author(s) (or their employer(s) unless otherwise stated in the text of the article) 2017. All rights reserved. No commercial use is permitted unless otherwise expressly granted.

\section{REFERENCES}

1. Pellino IM, Pellino G. Consequences of defensive medicine, second victims, and clinical-judicial syndrome on surgeons' medical practice and on health service. Updates Surg 2015;67:331-7.

2. Vandersteegen T, Marneffe W, Cleemput I, et al. The determinants of defensive medicine practices in Belgium. Health Econ Policy Law 2017;12:363-86.

3. US Congress Office of Technology Asessment. Defensive medicine and medical malpractice. Washington, DC: US Government Printing Office, 1994.
4. Panella M, Rinaldi C, Leigheb F, et al. Prevalence and costs of defensive medicine: a national survey of Italian physicians. $J$ Health Serv Res Policy 2017:211-7.

5. Studdert DM, Mello MM, Sage WM, et al. Defensive medicine among high-risk specialist physicians in a volatile malpractice environment. JAMA 2005;293:2609-17.

6. DeKay ML, Asch DA. Is the defensive use of diagnostic tests good for patients, or bad? Med Decis Making 1998;18:19-28.

7. Chawla A, Gunderman RB. Defensive medicine: prevalence, implications, and recommendations. Acad Radiol 2008;15:948-9.

8. Summerton N. Positive and negative factors in defensive medicine: a questionnaire study of general practitioners. BMJ 1995;310:27-9.

9. Bishop TF, Pesko M. Does defensive medicine protect doctors against malpractice claims? BMJ 2015;351:h5786.

10. Jena AB, Schoemaker L, Bhattacharya J, et al. Physician spending and subsequent risk of malpractice claims: observational study. BMJ 2015;351:h5516.

11. Asher E, Greenberg-Dotan S, Halevy J, et al. Defensive medicine in Israel: a nationwide survey. PLoS One 2012;7:e42613.

12. Hiyama T, Yoshihara M, Tanaka S, et al. Defensive medicine practices among gastroenterologists in Japan. World $\mathrm{J}$ Gastroenterol 2006;12:7671-5.

13. Kessler DP, Summerton N, Graham JR. Effects of the medical liability system in Australia, the UK, and the USA. Lancet 2006;368:240-6.

14. Ortashi O, Virdee J, Hassan R, et al. The practice of defensive medicine among hospital doctors in the United Kingdom. BMC Med Ethics 2013;14:42.

15. Reschovsky JD, Saiontz-Martinez CB. Malpractice claim fears and the costs of treating medicare patients: a new approach to estimating the costs of defensive medicine. Health Serv Res 2017.

16. Styrelsen for Patientsikkerhed, [Danish Patient Safety Authority]. Lov om autorisation af sundhedspersoner og om sundhedsfaglig virksomhed. 2011 https://stps.dk/da/om-os/love-og-regler/ patientklagecentret/oftest-benyttede-love-i-patientklagecenteret/ lov-om-autorisation-af-sundhedspersoner-og-om-sundhedsfagligvirksomhed/

17. Sandelowski M. Whatever happened to qualitative description? Res Nurs Health 2000;23:334-40.

18. Liamputtong P. Focus group methodology: principle and practice. California, USA: Sage Publications Limited, 2011.

19. Vermeire E, Van Royen P, Griffiths F, et al. The critical appraisal of focus group research articles. Eur J Gen Pract 2002;8:104-8.

20. Pedersen KM, Andersen JS, Søndergaard J. General practice and primary health care in Denmark. J Am Board Fam Med 2012;25 Suppl 1 (Suppl 1):S34-S38.

21. Malterud K, Siersma VD, Guassora AD. Sample size in qualitative interview studies: guided by information power. Qual Health Res 2015.

22. Bernard H, Ryan G. Analyzing qualitative data: systematic approaches. California, USA: Sage Publications, 2009.

23. Summerton $\mathrm{N}$. Trends in negative defensive medicine within general practice. Br J Gen Pract 2000;50:565-6.

24. Panella M, Rinaldi C, Leigheb F, et al. The determinants of defensive medicine in Italian hospitals: the impact of being a second victim. Rev Calid Asist 2016;31(Suppl 2):20-5.

25. Watt I, Nettleton S, Burrows R. The views of doctors on their working lives: a qualitative study. J R Soc Med 2008;101:592-7.

26. Nettleton S, Burrows R, Watt I. Regulating medical bodies? The consequences of the 'modernisation' of the NHS and the disembodiment of clinical knowledge. Sociol Health IIIn 2008;30:333-48.

27. Fairhurst K, May C. What general practitioners find satisfying in their work: implications for health care system reform. Ann Fam Med 2006;4:500-5.

28. Balint M. The doctor, his patient and the illness. London: Pitman Medical, 1957.

29. Cassell EJ. Doctoring: the nature of primary care medicine. Oxford: Oxford University Press, 1997.

30. Mead N, Bower P. Patient-centredness: a conceptual framework and review of the empirical literature. Soc Sci Med 2000;51:1087-110.

31. Fang $\mathrm{H}$, Miller NH, Rizzo J, et al. Demanding customers: consumerist patients and quality of care. B E J Econom Anal Policy 2011;11.

32. Tousijn W. Beyond decline: consumerism, managerialism and the need for a new medical professionalism. Health Sociology Review 2006;15:469-80.

33. Andersen MK, Pedersen LB, Dupont M, et al. General practitioners' attitudes towards and experiences with referrals due to supplemental health insurance. Fam Pract 2017;34:581-6.

34. Rowe M. Doctors' responses to medical errors. Crit Rev Oncol Hematol 2004:52:147-63. 
35. Newman MC. The emotional impact of mistakes on family physicians. Arch Fam Med 1996;5:71-5.

36. Christensen JF, Levinson W, Dunn PM. The heart of darkness: the impact of perceived mistakes on physicians. J Gen Intern Med 1992;7:424-31.

37. Allsop J, Mulcahy L. Maintaining professional identity: doctors' responses to complaints. Soc Health and IIIness 1998;20:802-24.
38. Wu AW. Medical error: the second victim. The doctor who makes the mistake needs help too. BMJ 2000;320:726-7.

39. Seys D, Wu AW, Van Gerven E, et al. Health care professionals as second victims after adverse events: a systematic review. Eval Health Prof 2013;36:135-62.

40. Malhotra A, Maughan D, Ansell J, et al. Choosing wisely in the UK: the academy of medical royal colleges' initiative to reduce the harms of too much medicine. BMJ 2015;350:h2308. 